\title{
HÁBITAT, ACTIVIDAD DIURNA Y MORFOMETRÍA DE CUATRO ESPECIES DE OFIDIOS (REPTILIA: SQUAMATA) DE CUBA
}

\author{
Josefina Blanco Ojeda y Ansel Fong G. \\ Centro Oriental de Ecosistemas y Biodiversidad (BIOECO), Museo de Historia Natural "Tomás Romay", \\ Enramadas \# 601, Santiago de Cuba 90100, Cuba \\ jade@bioeco.ciges.inf.cu, ansel@bioeco.ciges.inf.cu
}

\section{RESUMEN}

Los ofidios constituyen el segundo grupo en importancia dentro de los reptiles en las Antillas, pero la ecología de sus especies no ha sido muy estudiada. El propósito de este trabajo es dar a conocer algunos aspectos de la historia natural de cuatro especies de ofidios de Cuba (familias Dipsadidae y Tropidophiidae), acumulados durante ocho años de observación en 27 localidades y 11 tipos de hábitats. Las cuatro especies viven en una amplia diversidad de hábitats, incluyendo algunos con alta influencia humana. La especie de menor plasticidad ecológica fue Tropidophis wrighti, la que se encontró solo en el $45.4 \%$ de los hábitats y tuvo menor tolerancia por los ambientes antropizados. Todas las especies usan mayormente microhábitats terrestres, pero Cubophis cantherigerus y T. melanurus utilizan, en alguna medida, los substratos arbóreos. Dos especies (las de la familia Dipsadidae) tienen actividad diurna y parecen ser heliófilas, mientras que las otras dos (las de la familia Tropidophiidae) parecen tener poca actividad diurna y pueden considerarse como especies umbrófilas. Se detectaron dos agrupaciones morfológicas que parecen tener bases filogenéticas, ya que están poco relacionadas con el microhábitat o la alimentación.

Palabras clave: Antillas, agrupaciones morfológicas, hábitat, historia natural, serpientes.

\section{ABSTRACT}

Snakes are the second most diverse reptile group in the West Indies, however the ecology of these species have been rarely studied so far. Our purpose is to publish some aspects of the natural history of four Cuban ophidians (families Dipsadidae and Tropidophiidae), gather together along eight years and observations at 27 localities and 11 habitat types. Every species used most of the habitats, including some human-altered types; the exception was Tropidophis wrighti, living only in the $45.4 \%$ of the habitats, using altered environments in less proportion. The four snakes were found on terrestrial microhabitats, only Cubophis cantherigerus and T. melanurus were sometimes found in bushes and trees. Two species (family Dipsadidae) are diurnal and sun lovers, the other species (family Tropidophiidae) had little diurnal activity and are considered herein as shade lovers. We detected two morphological groups unrelated to microhabitat or diet of these snakes, and appearing to have phylogenetic bases.

Key words: habitat, morphological groups, natural history, snakes, West Indies.

\section{INTRODUCCIÓN}

Los ofidios constituyen el segundo grupo en importancia dentro de los reptiles (con 3,315 especies descritas, según Uetz et al., 2011), proporción que se repite en Las Antillas, donde habitan unas 148 especies (Hedges, 2009; Henderson y Powell, 2009) y aún otras se encuentran en proceso de descripción. Sin embargo, la fauna de serpientes de las Antillas ha sido prácticamente ignorada en cuanto a su ecología y solo se han realizado unos pocos estudios, en especial con especies de la familia Boidae (Henderson y Powell, 1999). En el 62\% de las especies no se han 
realizado investigaciones detalladas y no existe ninguna información sobre la historia natural de muchos de los taxones (Henderson y Powell, 2009).

Las especies de Cuba han recibido una mayor atención desde el punto de vista taxonómico (la cronología de estos trabajos se describe en Henderson y Powell, 1999). Por el contrario, las publicaciones dirigidas solo al estudio de la biología de ofidios son aún escasas (exceptuando a la familia Boidae) y se refieren principalmente a la cría en cautiverio y los parásitos, así como algunas notas que abordan la dieta, los cambios de coloración y la reproducción, entre otros pocos aspectos (ver la revisión completa en Henderson y Powell, 2009). Henderson y Crother (1989) y Henderson y Sajdak (1996) incluyeron datos sobre las especies cubanas en sus trabajos sobre alimentación de colúbridos antillanos. Schwartz y Henderson (1991) reunieron toda la información existente de cada una de las especies conocidas en aquel momento, incorporando sus datos y observaciones fortuitas, información que fue actualizada y ampliada por Henderson y Powell (2009).

Muchos de los datos publicados sobre los ofidios de Cuba se basan en observaciones anecdóticas y son mayormente descripciones cualitativas casuales que no incluyen datos cuantitativos, generalmente incluidos en trabajos taxonómicos, listas y catálogos. Con este trabajo nos proponemos dar a conocer algunos aspectos de la historia natural de cuatro especies de ofidios cubanos como forma de contribuir al conocimiento de estos pocos estudiados reptiles, utilizando datos acumulados durante ocho años de observación.

\section{OBJETIVOS}

- Cuantificar el uso de diferentes tipos de hábitats por cuatro especies de ofidios cubanos.

- Determinar los horarios y tipos de actividad diurna de estas especies de ofidios.

- Explorar la existencia de agrupaciones a partir de los datos morfométricos de las especies.

\section{MATERIALES Y MÉTODOS}

Se tomaron datos a los ofidios detectados en estudios y muestreos de campo realizados por los autores y colaboradores durante los años 1995-2003 en 27 localidades de Cuba. Las observaciones se hicieron en áreas boscosas (Bosque pluvial esclerófilo, Bosque siempreverde, Bosque semideciduo, Complejo de vegetación sobre mogotes), en matorrales (Charrascal, Matorral xeromorfo), en plantaciones (Pinar, Cafetal, Pastizal), en vegetación secundaria y dentro de pueblos y ciudades. Las características de cada uno de estos hábitats se describen en Reyes (2007).

Se anotaron los siguientes datos: especie, hora de observación, estadío etáreo (adulto/juvenil), sexo (cuando pudo determinarse), hábitat (nombrados anteriormente), microhábitat (suelo, bajo roca o tronco caído, rama/tronco de árbol o arbusto, río/arroyo, otros), altura sobre el suelo (en metros), actividad en el momento de la observación (asoleándose, alimentándose, moviéndose, forrajeando, inactivo), iluminación (sol directo, sol filtrado, sombra), longitud hocico-cloaca (en milímetros), longitud de la cola (en milímetros, solo en individuos con colas completas) y peso (en gramos). No todos los datos pudieron ser obtenidos de cada individuo.

Algunos ejemplares fueron colectados como material de referencia y se depositaron en la colección herpetológica del Centro Oriental de Ecosistemas y Biodiversidad (BIOECO) en Santiago de Cuba. Para la comparación entre promedios se utilizaron las pruebas U de MannWhitney y Kruskal-Wallis (Sokal y Rohlf, 1995) teniendo en cuenta que los datos no se ajustaron a una distribución normal. 


\section{RESULTADOS Y DISCUSIÓN}

Se obtuvieron datos de 108 ejemplares de cuatro especies de las familias Dipsadidae y Tropidophiidae. La especie con el menor número de observaciones fue Tropidophis wrighti $(\mathrm{N}=10)$. Las otras tres especies fueron vistas en proporciones similares: Tropidophis melanurus $\mathrm{N}=34$, Caraiba andreae $\mathrm{N}=32$, Cubophis cantherigerus $\mathrm{N}=32$. A continuación exponemos los datos de cada especie por separado.

\section{Caraiba andreae}

Esta especie se encontró en ocho hábitats (fig. 1), con el 50\% de los ejemplares observados en bosques. También las plantaciones y la vegetación secundaria presentaron porcentajes altos de utilización, mientras que en zonas antropizadas solo se vio un individuo (fig. 1). La mayoría de los ejemplares de esta especie se encontró en el suelo (83.9\% del total visto) o bajo rocas (9.7\%), solo dos individuos fueron vistos en tocones (fig. 2) a alturas menores de $70 \mathrm{~cm}$.

El mayor porcentaje de los ejemplares se encontró en actividad en el momento de su observación, predominando los animales que se detectaron asoleándose, seguidos de los ejemplares forrajeando, moviéndose y alimentándose (Tabla 1). Considerando solo los animales activos, más de la mitad se observó al sol directo o filtrado, mientras que el resto se vio a la sombra (Tabla 2). Todas las serpientes se observaron entre las 08:00 h y las 17:00 h, aunque los ejemplares activos solo se vieron entre las 09:00 h y las 15:00 h. El número de individuos activos aumentó a medida que transcurrió el día para alcanzar el valor máximo alrededor del mediodía y disminuir a partir de ese momento (fig. 3).

La longitud y el peso de 16 ejemplares adultos se muestran en la Tabla 3. No se tomaron datos a ejemplares juveniles, solo un macho sub-adulto que midió $125 \mathrm{~mm}$ de longitud hocicocloaca y $72 \mathrm{~mm}$ de longitud de la cola.

\section{Cubophis cantherigerus}

Se observaron ejemplares en nueve hábitats diferentes (fig. 1). La mayor cantidad de individuos se vio en bosques y vegetación secundaria, aunque también en matorrales se observó un alto número. La menor cantidad se encontró en áreas antropizadas (fig. 1). De los cinco tipos de microhábitats donde se observó esta especie, el más utilizado fue el suelo con más de la mitad de las observaciones (fig. 2). También se vieron individuos bajo piedras y troncos caídos en proporción relativamente alta, al igual que en ramas y troncos de árboles o arbustos. Un solo ejemplar se vio nadando en un arroyo y otro dentro de una bromelia, siendo estos los microhábitats menos utilizados. Los ejemplares vistos en microhábitats arbustivos estuvieron en alturas de entre 0.5 y $5.0 \mathrm{~m}$.

Solamente un tercio de los individuos observados estaban inactivos, la mayoría de los ejemplares activos se encontró asolándose o forrajeando (Tabla 1). De los ejemplares activos, la mayor parte estuvo al sol directo o filtrado, el $42.1 \%$ restante estaba a la sombra (Tabla 2). Lo más temprano que se observó un ejemplar fue a las 08:15 h y lo más tarde a las 17:25 h, aunque las 17:08 fue la hora más avanzada a la que se vio una serpiente activa (fig. 3). La mayor actividad diurna se desarrolló alrededor de las 09:00 h, aunque se observaron individuos activos durante el resto de las horas del día (fig. 3). Los datos morfométricos de nueve ejemplares adultos y cinco juveniles se muestran en la Tabla 3.

\section{Tropidophis melanurus}

Los ejemplares de esta especie estuvieron presentes en siete hábitats (fig. 1), fundamentalmente en vegetación secundaria y matorrales, aunque en bosques también se observó un número relativamente alto de individuos (fig. 1). La mayoría de los ejemplares estaban en el suelo (29.4\% 
del total) o bajo piedras y troncos (38.2\%), ocho animales se encontraron en troncos de árboles o arbustos (fig. 2) a alturas de entre 0.4 y $2.2 \mathrm{~m}$. Tres individuos fueron vistos semi-sumergidos en el agua de arroyos (fig. 2).

Casi dos tercios de las serpientes detectadas estaban inactivas (Tabla 1), la mayoría de las activas estaba forrajeando $(29.0 \%$ del total) y solo tres ejemplares fueron vistos asoleándose y alimentándose (Tabla 1). De los ejemplares activos, el 63.6\% se encontró a la sombra y solo uno se observó al sol directo (Tabla 2). Las observaciones individuales se realizaron entre las 06:10 h y las 17:10 h, pero ejemplares activos solo se vieron entre las 08:15 h y las 14:10 h. El número de individuos activos de T. melanurus fue mayor alrededor de las 08:00 h, disminuyendo a medida que transcurrió el día (fig. 3). Los datos morfométricos de 25 ejemplares adultos se muestran en la Tabla 3.

\section{Tropidophis wrighti}

Esta especie se observó en cinco tipos de hábitats (fig. 1), el 44.4\% de los ejemplares se encontraron en bosques, dos individuos se observaron en matorrales, dos en plantaciones (pinares) y solo uno en vegetación secundaria (fig. 1). Los ejemplares de esta especie se observaron a alturas de entre 0.15 y $0.60 \mathrm{~m}$, bajo rocas, debajo de la corteza de árboles y un ejemplar debajo de la corteza de un tronco seco sobre el suelo (fig. 2).

Todos los ejemplares estaban inactivos en el momento de su observación (Tabla 1) y a la sombra. Lo más temprano que se observó un animal fue a las 07:00 h y lo más tarde a las 17:00 h. Los datos morfométricos de nueve ejemplares adultos se muestran en la Tabla 3.

Las cuatro especies viven en una amplia diversidad de hábitats, $T$. wrighti fue la especie de menor plasticidad ecológica pues se encontró en menos de la mitad de los hábitats considerados (fig. 1). El resto de las especies vive en más del $60 \%$ de los hábitats muestreados, con el valor máximo para $C$. cantherigerus $(81.8 \%)$ lo cual confirma la amplia plasticidad ecológica mencionada por Fong y Viña (1998). La mayoría de las especies son capaces de vivir en hábitats con influencia humana (plantaciones, vegetación secundaria, pueblos y ciudades), pero solo tres ejemplares de $T$. wrighti se encontraron en vegetaciones no naturales (fig. 1), incluyendo una plantación de pinos con características estructurales similares a las de un bosque primario, lo que indicaría un mayor uso de hábitats naturales.

Todas las especies usan mayormente microhábitats terrestres, encontrándose directamente sobre el suelo o refugiadas bajo rocas o troncos caídos (fig. 2). Dos especies, C. cantherigerus y $T$. melanurus, utilizan en alguna medida los substratos arbóreos. La primera se encuentra a mayores alturas (promedio $=1.79 \mathrm{~m}, 0.5-5.0 \mathrm{~m}$ ) que la segunda (promedio=1.22 $\mathrm{m}, 0.4-2.2 \mathrm{~m}$ ), pero T. melanurus usa estos microhábitats en mayor proporción (el 23.5\% de los ejemplares en arbustos o árboles vs. el 15.6\% en C. cantherigerus). Solo dos ejemplares de cada una de las otras especies se observaron en sitios no terrestres, siempre a alturas menores de $0.70 \mathrm{~m}$. En general, estos resultados coinciden con las observaciones realizadas por Schwartz y Henderson (1991) y por Henderson y Powell (2009) para cada especie.

La mayoría de los dipsádidos se observaron en actividad, predominado los animales asoleándose (Tabla 1), mientras que la mayor parte de los ejemplares de la familia Tropidophiidae estaban inactivos al momento de detectarlos (Tabla 1). Teniendo en cuenta la proporción de animales encontrados en actividad durante las horas del día (entre las 08:00 y las 18:00 h) puede considerarse la existencia de dos especies con actividad diurna y una especie con poca actividad diurna. La mayoría de los ejemplares de Dipsadidae estaban activos durante las horas del día, mientras que la mayoría de los individuos de T. melanurus estaban inactivos (fig. 3). Todos los 
ejemplares de T. wrighti estaban inactivos, por lo que parece ser una especie nocturna (fig. 3). Estos resultados concuerdan con lo expuesto por Schwartz y Henderson (1991) y por Henderson y Powell (2009) para estas especies y por Fong (2001) para C. cantherigerus.

Encontramos diferencias en los horarios de actividad diurna de las dos especies de Dipsadidae: los ejemplares de $C$. andreae tienen mayor actividad alrededor del mediodía, mientras que los de $C$. cantherigerus se encuentran más activos hacia las horas medias de la mañana (aunque mantienen un nivel de actividad relativamente alto todo el día) (fig. 3). Esto podría indicar una separación en el nicho temporal para tratar de disminuir la competencia entre las dos especies. La mayor actividad de T. melanurus en las primeras horas de la mañana y su desaparición en la tarde (fig. 3), pudieran indicar el final de un período de actividad nocturna que se extiende hasta las primeras horas de la mañana.

Ya que no se realizaron muestreos en horas de la noche, no podemos asegurar que las especies en que la mayoría de los ejemplares se encontraron inactivos durante el día indiquen necesariamente que son nocturnas, aunque es una posibilidad a considerar. Igualmente, las especies con actividad diurna pudieran tener también actividad durante la noche, pero no hicimos muestreos nocturnos para corroborarlo (aunque en otros estudios hemos observado individuos de T. melanurus forrajeando alrededor de las 21:45 h). E1 horario de actividad de cada especie es uno de los aspectos que necesita mayor estudio en el futuro, con muestreos uniformados en todas las horas del día y un mayor tamaño de muestra que permitan confirmar las hipótesis formuladas.

Coincidiendo con los horarios de actividad, los dos dipsádidos parecen aprovechar y preferir la luz del sol, pues más de la mitad de los ejemplares en actividad estaban al sol directo o filtrado (Tabla 2) y muchos de ellos estaban asoleándose (Tabla 1). Por el contrario, solo dos ejemplares de T. melanurus estaban asoleándose (Tabla 2), mientras que todos los T. wrighti estuvieron a la sombra. Según estos resultados, podría considerarse a las especies de Caraiba y Cubophis como heliófilas y a las dos especies de Tropidophis como umbrófilas.

Los datos morfométricos de nuestros ejemplares (Tabla 3) se encuentran dentro de los valores registrados en los trabajos taxonómicos de otros autores. No encontramos diferencias significativas $(\mathrm{p}>0.05)$ entre las mediciones de los sexos de T. melanurus (Longitud hocicocloaca: $U=81.00$, longitud de la cola: $U=96.00$, peso: $U=49.00$ ) y de $T$. wrighti (Longitud hocicocloaca: $U=13.00$, longitud de la cola: $U=14.50$ ).

Se apreciaron diferencias significativas entre las especies en la relación longitud de la cola/longitud total $(\mathrm{H}=44.53, \mathrm{p}<0.0001)$ y peso/longitud total $(\mathrm{H}=7.46, \mathrm{p}<0.05)$, sugiriendo la separación en dos grupos, uno formado por las especies de la familia Tropidophiidae (con colas cortas y tendencia a ser más robustos) y el otro por las de Dipsadidae (con colas más largas y menos pesados) (fig. 4). Estas agrupaciones no parecen estar relacionadas directamente con el microhábitat utilizado ni con la alimentación de estas especies, la que es básicamente similar (Henderson y Crother, 1989; Henderson y Powell, 2009), pero otros aspectos ecológicos no estudiados pudieran revelar alguna afinidad.

Por tanto, no detectamos agrupaciones morfo-ecológicas como las existentes en determinadas comunidades de ofidios continentales (Guyer y Donnelly, 1990), al menos dentro de estas cuatro especies, pero estos resultados deben ser confirmados analizando un mayor número de taxones, especialmente especies simpátricas. Las diferencias obtenidas podrían deberse a aspectos filogenéticos más que a causas ecológicas, como se ha propuesto para otras comunidades de serpientes neotropicales (Vitt y Vangilder, 1983; Guyer y Donnelly, 1990). 
Tabla 1. Número (porcentaje) de individuos de cuatro especies de ofidios de Cuba observados en diferentes tipos de actividad.

\begin{tabular}{|llllll|}
\hline \multicolumn{1}{|c}{ Especie } & Asoleándose & Alimentándose & Moviéndose & Forrajeando & Inactivos \\
\hline Caraiba andreae & $6(27.3)$ & $3(13.6)$ & $4(18.1)$ & $4(18.1)$ & $5(22.7)$ \\
Cubophis cantherigerus & $9(31.0)$ & $1(3.5)$ & $2(6.9)$ & $7(24.1)$ & $10(34.5)$ \\
Tropidophis melanurus & $2(6.4)$ & $1(3.2)$ & 0 & $9(29.0)$ & $19(61.3)$ \\
Tropidophis wrighti & 0 & 0 & 0 & 0 & $7(100.0)$ \\
\hline
\end{tabular}

Tabla 2. Número (porcentaje) de individuos de tres especies de ofidios de Cuba detectados bajo diferentes categorías de iluminación (considerando solo los individuos observados en actividad).

\begin{tabular}{|llll|}
\hline Especie & Sol directo & Sol filtrado & Sombra \\
\hline Caraiba andreae & $8(50.0 \%)$ & $1(6.2 \%)$ & $7(43.8 \%)$ \\
Cubophis cantherigerus & $8(42.1 \%)$ & $3(15.8 \%)$ & $8(42.1 \%)$ \\
Tropidophis melanurus & $1(9.1 \%)$ & $3(27.3 \%)$ & $7(63.6 \%)$ \\
\hline
\end{tabular}

Tabla 3. Datos morfométricos de cuatro especies de ofidios de Cuba. Se muestra la mediana, entre paréntesis los valores mínimos-máximos y debajo el número de ejemplares utilizados. $\mathrm{LHC}=$ Longitud hocico-cloaca, $\mathrm{LC}=$ Longitud de la cola, $\mathrm{P}=$ Peso.

\begin{tabular}{|c|c|c|c|c|}
\hline Especie & Estadio/sexo & LHC (mm) & $\mathrm{LC}(\mathrm{mm})$ & $P(g)$ \\
\hline \multirow[t]{2}{*}{ Caraiba andreae } & Adultos & $396(302-465)$ & $208(160-275)$ & $38.5(26.0-48.0)$ \\
\hline & & $\mathrm{N}=16$ & $\mathrm{~N}=12$ & $\mathrm{~N}=6$ \\
\hline \multirow[t]{4}{*}{ Cubophis cantherigerus } & Adultos & $700(601-820)$ & $270(78-333)$ & $122.2(77.3-214.9)$ \\
\hline & & $\mathrm{N}=9$ & $\mathrm{~N}=9$ & $\mathrm{~N}=8$ \\
\hline & Juveniles & $262(106-300)$ & $103(46-105)$ & $8.5(7.0-15.0)$ \\
\hline & & $\mathrm{N}=5$ & $\mathrm{~N}=4$ & $\mathrm{~N}=4$ \\
\hline \multirow[t]{4}{*}{ Tropidophis melanurus } & Machos & $520(396-714)$ & $70(39-105)$ & $83.1(24.7-185.0)$ \\
\hline & & $\mathrm{N}=16$ & $\mathrm{~N}=16$ & $\mathrm{~N}=12$ \\
\hline & Hembras & 478 (314-690) & $60(46-82)$ & $81.1(39.0-185.0)$ \\
\hline & & $\mathrm{N}=9$ & $\mathrm{~N}=9$ & $\mathrm{~N}=8$ \\
\hline \multirow[t]{4}{*}{ Tropidophis wrighti } & Machos & $276(272-375)$ & $49(42-52)$ & 14.0 \\
\hline & & $\mathrm{N}=3$ & $\mathrm{~N}=3$ & $\mathrm{~N}=1$ \\
\hline & Hembras & $362(293-385)$ & $40(33-50)$ & 17.0 \\
\hline & & $\mathrm{N}=6$ & $\mathrm{~N}=6$ & $\mathrm{~N}=1$ \\
\hline
\end{tabular}


Cubophis cantherigerus $(\mathrm{n}=32)$

\begin{tabular}{lrrr}
1 & 3 & 3 & 2 \\
\hline
\end{tabular}
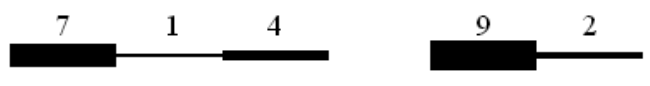

Caraiba andreae $(\mathrm{n}=32)$

\begin{tabular}{|c|c|c|c|c|c|c|c|c|c|c|}
\hline PS & SV & $\mathrm{SD}$ & $\mathrm{CV}$ & $\mathrm{CH}$ & XR & $\mathrm{PN}$ & $\mathrm{CF}$ & PT & VS & AN \\
\hline
\end{tabular}

Tropidophis melanurus $(\mathrm{n}=34)$

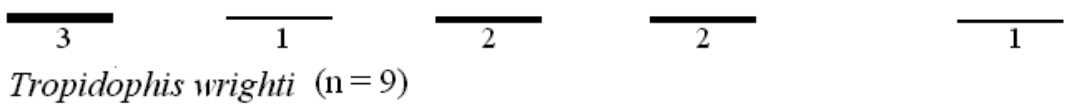

Figura 1. Distribución por hábitats de cuatro especies de ofidios de Cuba. El grosor de las líneas es proporcional al número de ejemplares visto en cada hábitat (mostrado contiguo a las líneas). $\mathrm{PS}=$ Pluvisilva, $\mathrm{SV}=\mathrm{Bosque}$ siempreverde, $\mathrm{SD}=\mathrm{B}$ osque semideciduo, $\mathrm{CV}=$ Complejo de vegetación sobre mogotes, $\mathrm{CH}=$ Charrascal, $\mathrm{XR}=$ Matorral xeromorfo, $\mathrm{PN}=\mathrm{Pinar}, \mathrm{CF}=\mathrm{Cafetal}, \mathrm{PT}=$ Pastizal, VS= Vegetación secundaria, AN= Pueblos y ciudades.

Cubophis cantherigerus $(\mathrm{n}=32)$

\begin{tabular}{lllll}
19 & 6 & 5 & 1 & 1 \\
\hline
\end{tabular}

Caraiba andreae $(\mathrm{n}=31)$

26 3

2

\begin{tabular}{|l|l|l|l|l|}
\hline Suelo & Bajo rocas & Arbóreos & En el agua & Otros \\
\hline
\end{tabular}

10

13

8

3

Tropidophis melanurus $(\mathrm{n}=34)$

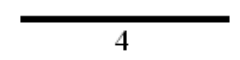

Tropidophis wrighti $(\mathrm{n}=7)$

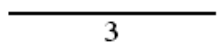

Figura 2. Distribución por microhábitats de cuatro especies de ofidios de Cuba. El grosor de las líneas es proporcional al número de ejemplares visto en cada microhábitat (mostrado contiguo a las líneas). "Otros" incluye ejemplares vistos bajo corteza, en tocones y dentro de bromeliáceas. 

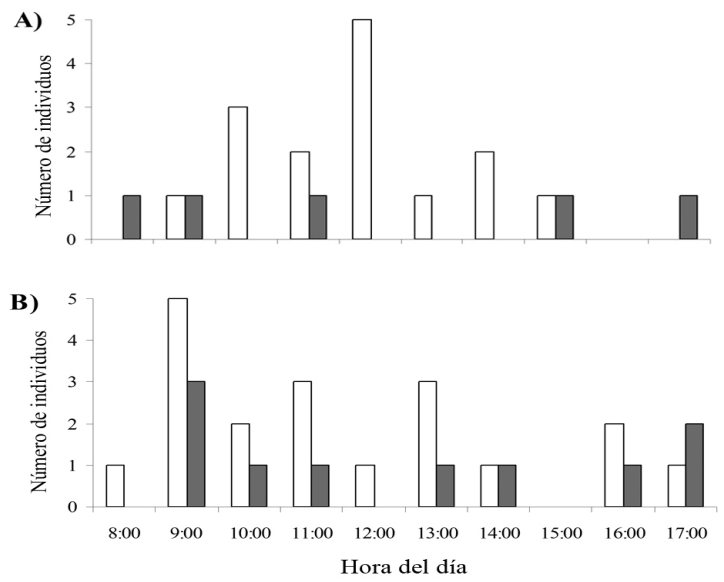

C)

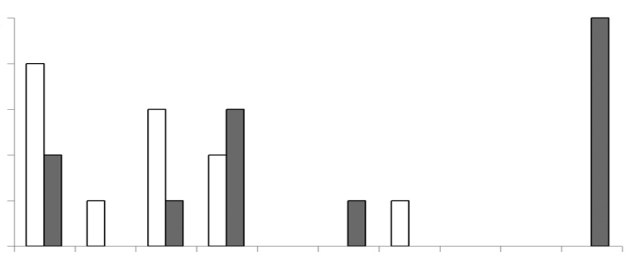

D)

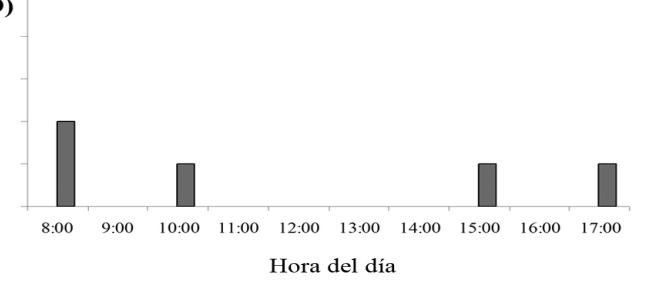

Figura 3. Número de individuos de cuatro especies de ofidios observados durante las horas del día en actividad (barras blancas) o inactivos (barras oscuras). Los ejemplares activos incluyen aquellos vistos forrajeando, alimentándose, asoleándose o en movimiento (ver Tabla 1). Caraiba andreae (A), Cubophis cantherigerus (B), Tropidophis melanurus (C), Tropidophis wrighti (D).
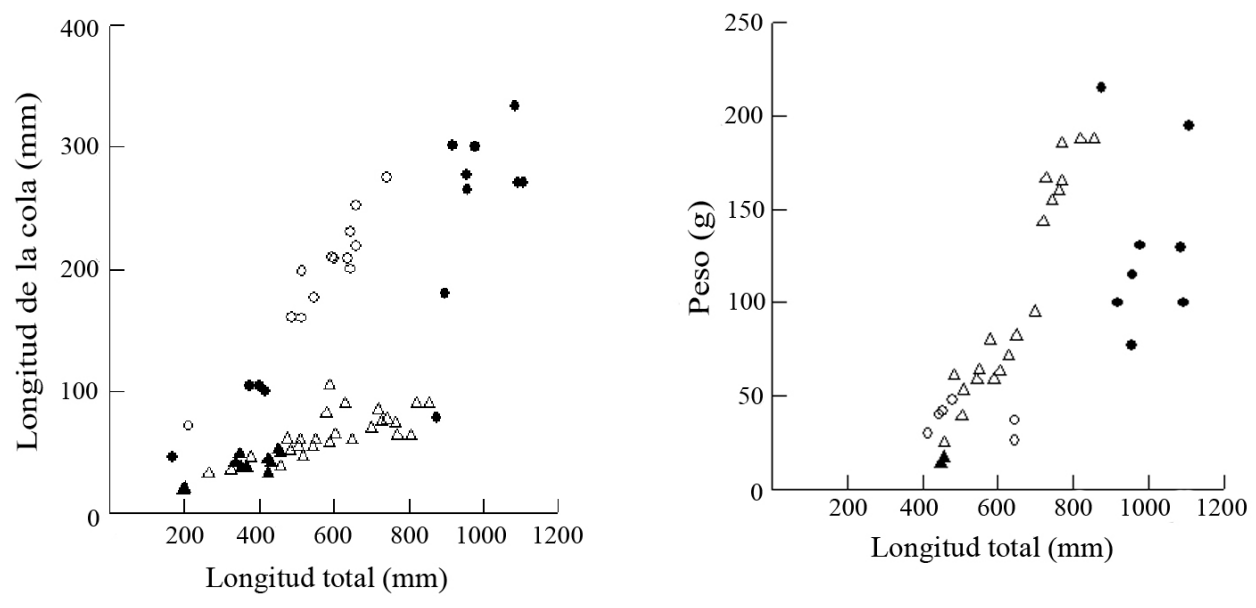

Figura 4. Relación entre la longitud de la cola, la longitud total y el peso en cuatro especies de ofidios de Cuba: Caraiba andreae (círculos blancos), Cubophis cantherigerus (círculos negros), Tropidophis melanurus (triángulos blancos), Tropidophis wrighti (triángulos negros).

\section{AGRADECIMIENTOS}

Rolando Viña, David Maceira, Beatriz Lauranzón, Rolando Teruel, María del Carmen Fagilde y Pedro López aportaron datos y ejemplares que contribuyeron al aumento de nuestra muestra. Robert W. Henderson envió valiosa literatura. Dos revisores hicieron útiles comentarios a la versión inicial del manuscrito.

\section{LITERATURA CITADA}

Fong, A. 2001. Características fundamentales de la herpetofauna del macizo montañoso Sagua-

Baracoa, Cuba. Boletín Soc. Herp. Mexicana, 9 (1): 23-28. 
Fong, A. y N. Viña. 1998. Reptiles de Nipe-Sagua-Baracoa, pp. 678-712. En: BIOECO (ed.), Diversidad Biológica del Macizo Montañoso Nipe-Sagua-Baracoa. Informe Parcial. Centro Oriental de Ecosistemas y Biodiversidad (BIOECO), Santiago de Cuba.

Guyer, C. y M. Donnelly. 1990. Length-mass relationships among an assemblage of tropical snakes in Costa Rica. J. Trop. Ecol., 6: 65-76.

Hedges, S. B. 2009. Caribherp: database of West Indian amphibians and reptiles (http://evo. bio.psu.edu/caribherp/). Pennsylvania State University, University Park. [Revisado el 26 Noviembre 2009].

Henderson, R. W. y B. I. Crother. 1989. Biogeographic patterns of predation in West Indian colubrid snakes, pp. 479-517. En: C. A. Woods (ed.) Biogeography of the West Indies. Past, present and future. Sandhill Crane Press, Gainesville.

Henderson, R. W. y R. Powell. 1999. West Indian Herpetoecology, pp. 223-268. En: B. I. Crother (ed.) Caribbean Amphibians and Reptiles. Academic Press, California.

Henderson, R. W. y R. Powell. 2009. Natural history of West Indian reptiles and amphibians. University Press of Florida, Gainesville, xxiv +496 pp.

Henderson, R. W. y R. A. Sajdak. 1996. Diets of West Indian racers (Colubridae: Alsophis): Composition and biogeographic implications, pp. 317-326. En: R. Powell y R. W. Henderson (eds.) Contributions to West Indian Herpetology: A Tribute to Albert Schwartz. Contributions to Herpetology, volume 12. Ithaca, N. Y.

Reyes, O. J. 2007. Clasificación de la vegetación de la Región Oriental de Cuba. Revista del Jardín Botánico Nacional de Cuba [En prensa].

Schwartz, A. y R. W. Henderson. 1991. Amphibians and reptiles of the West Indies. Descriptions, distributions, and natural history. University of Florida Press, Gainesville, xvi +720 pp.

Sokal, R. R. y F. J. Rohlf. 1995. Biometry: The principles and practice of statistic in biological research. W. H. Freeman, San Francisco, 887 pp.

Uetz, P., J. Hošek y J. Hallerman. 2011. The Reptile Database (http://www.reptile-database.org) [Revisado el 27 Enero 2011].

Vitt, L. J. y L. D. Vangilder. 1983. Ecology of a snake community in northeastern Brazil. Amphibia-Reptilia, 4: 273-296. 